\title{
molecules
}

ISSN 1420-3049

www.mdpi.com/journal/molecules

Article

\section{Plasma Pharmacokinetics of Polyphenols in a Traditional Japanese Medicine, Jumihaidokuto, Which Suppresses Propionibacterium acnes-Induced Dermatitis in Rats}

\section{Takashi Matsumoto ${ }^{1, *}$, Yousuke Matsubara ${ }^{1}$, Yasuharu Mizuhara ${ }^{1}$, Kyoji Sekiguchi ${ }^{1}$, Junichi Koseki ${ }^{1}$, Kazuaki Tsuchiya ${ }^{1}$, Hiroaki Nishimura ${ }^{2}$, Junko Watanabe ${ }^{1}$, Atsushi Kaneko ${ }^{1}$, Kazuya Maemura ${ }^{1}$, Tomohisa Hattori ${ }^{1}$ and Yoshio Kase ${ }^{1}$}

1 Tsumura Research Laboratories, Kampo Scientific Strategies Division, Tsumura \& Co., Ibaraki 300-1192, Japan; E-Mails: matsubara_yousuke@mail.tsumura.co.jp (Y.M.); mizuhara_yasuharu@mail.tsumura.co.jp (Y.M.); sekiguchi_kyouji@mail.tsumura.co.jp (K.S.); koseki_junichi@mail.tsumura.co.jp (J.K.); tsuchiya_kazuaki@mail.tsumura.co.jp (K.T.); watanabe_junko@mail.tsumura.co.jp (J.W.); kaneko_atsushi@mail.tsumura.co.jp (A.K.); maemura_kazuya@mail.tsumura.co.jp (K.M.); hattori_tomohisa@mail.tsumura.co.jp (T.H.); kase_yoshio@mail.tsumura.co.jp (Y.K.)

2 Kampo Formulations Development Center, Production Division, Tsumura \& Co., Ibaraki 300-1192, Japan; E-Mail: nishimura_hiroaki@mail.tsumura.co.jp

* Author to whom correspondence should be addressed;

E-Mail: matsumoto_takashi@mail.tsumura.co.jp; Tel.: +81-29-889-3852; Fax: +81-29-889-3870.

Academic Editor: Marcello Iriti

Received: 26 August 2015 / Accepted: 27 September 2015 / Published: 30 September 2015

\begin{abstract}
Most orally administered polyphenols are metabolized, with very little absorbed as aglycones and/or unchanged forms. Metabolic and pharmacokinetic studies are therefore necessary to understand the pharmacological mechanisms of polyphenols. Jumihaidokuto (JHT), a traditional Japanese medicine, has been used for treatment of skin diseases including inflammatory acne. Because JHT contains various types of bioactive polyphenols, our aim was to clarify the metabolism and pharmacokinetics of the polyphenols in JHT and identify active metabolites contributing to its antidermatitis effects. Orally administered JHT inhibited the increase in ear thickness in rats induced by intradermal injection of Propionibacterium acnes. Quantification by LC-MS/MS indicated that JHT contains various types of flavonoids and is also rich in hydrolysable tannins, such as 1,2,3,4,6-penta- $O$-galloyl glucose. Pharmacokinetic and antioxidant analyses showed that some flavonoid conjugates, such as genistein
\end{abstract}


7-O-glucuronide and liquiritigenin 7-O-glucuronide, appeared in rat plasma and had an activity to inhibit hydrogen peroxide-dependent oxidation. Furthermore, 4-O-methylgallic acid, a metabolite of Gallic acid, appeared in rat plasma and inhibited the nitric oxide reaction. JHT has numerous polyphenols; it inhibited dermatitis probably via the antioxidant effect of its metabolites. Our study is beneficial for understanding in vivo actions of orally administered polyphenol drugs.

Keywords: jumihaidokuto; flavonoid; tannin; genistein; liquiritigenin; Gallic acid; glucuronides; pharmacokinetics; antioxidant; dermatitis

\section{Introduction}

Most orally administered polyphenols are metabolized in a living body. Flavonoids are known to undergo conjugation in the intestine and liver and circulate predominantly as glucuronides and sulfates with very little as aglycones and/or unchanged forms [1-4]. Moreover, some of polyphenols with a sugar moiety, hydrolysable tannins, and others are hydrolyzed by intestinal bacteria and then absorbed in the intestines [1,5-8]. Therefore, it is important to elucidate the metabolism and plasma pharmacokinetics of polyphenols in a study focusing on diseases of the nondigestive organs such as skin. In addition, evaluating pharmacological benefits of the metabolites identified in the systemic circulation is necessary.

Jumihaidokuto (JHT), a pharmaceutical-grade traditional Japanese (kampo) medicine, has been used widely for treatment of skin symptoms such as reddening, swelling, sharp pain, burning sensation, and skin diseases including acute and/or purulent skin diseases, urticaria, eczema, and athlete's foot. JHT is reportedly effective in patients with inflammatory acne [9], and inhibited hapten-induced inflammation in a mouse model of allergic dermatitis [10]. We recently reported that JHT inhibited increases in ear thickness in two models of acute dermatitis developed using phorbol myristate acetate or Propionibacterium acnes (P. acnes), a gram-positive anaerobic microbe [11]. To investigate the potential mechanism of JHT, we examined antioxidant activities of the crude drugs and constituents, demonstrating that polyphenols originating from Quercus cortex (Q. cortex), Schizonepetae spica (S. spica), and Glycyrrhizae radix ( $G$. radix) were active in an assay focusing on antioxidant activity against reactive oxygen species (ROS).

Consistent with our previous study [11], we hypothesized that JHT ameliorates dermatitis through antioxidant actions by the polyphenols in JHT. In this study, for the first time, we examined plasma pharmacokinetics of polyphenols present in JHT and their metabolites, and identified active metabolites contributing to its antidermatitis effect.

\section{Results and Discussion}

\subsection{Antidermatitis Effect of JHT}

We first investigated whether JHT ameliorated P. acnes-induced dermatitis. Rats subjected to intradermal injection of $P$. acnes and orally administered distilled water developed rapid ear swelling with cutaneous erythema, followed by a $181 \%$ increase in ear thickness in a $24 \mathrm{~h}$ period compared with 
that prior to injection (Figure 1). On the other hand, rats treated with $0.5 \mathrm{~g} / \mathrm{kg}$ JHT exhibited only a $132 \%$ increase, whereas prednisolone (PDN) exhibited a $123 \%$ increase. These results suggest that JHT has a potential antidermatitis effect.

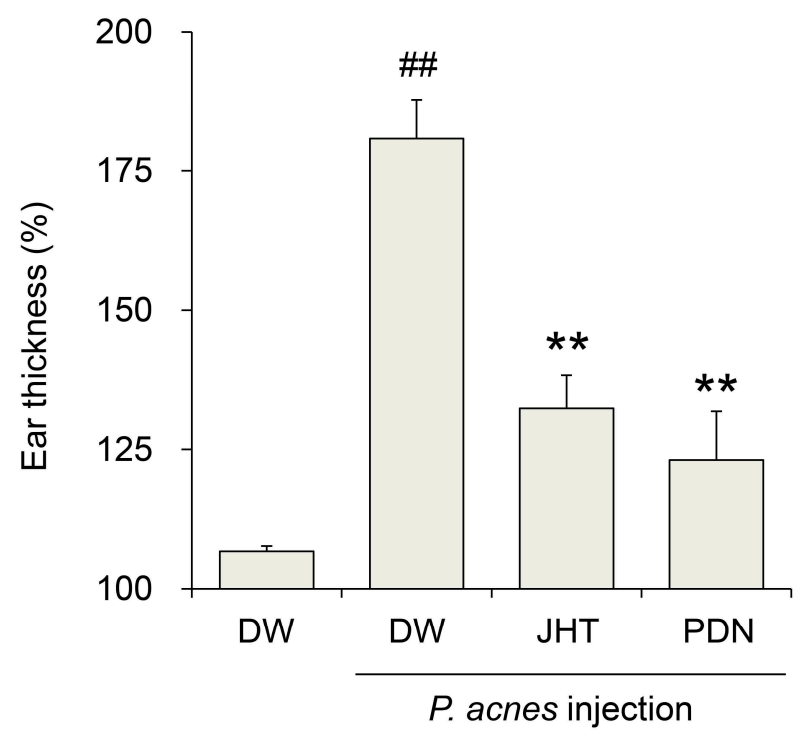

Figure 1. Suppression of Propionibacterium acnes (P. acnes)-induced dermatitis in rats by jumihaidokuto (JHT) administration. Dermatitis was induced in rats by injection of $P$. acnes $(0.14 \mathrm{mg} / 50 \mu \mathrm{L} /$ ear $)$ into the pinna. The ear thickness was measured at 0 and $24 \mathrm{~h}$ after the injection. JHT suspended in distilled water (DW) was administered orally at a dose of $0.5 \mathrm{~g} / 10 \mathrm{~mL} / \mathrm{kg}$ at $1 \mathrm{~h}$ before and $6 \mathrm{~h}$ after the injection. Prednisolone (PDN, $10 \mathrm{mg} / 10 \mathrm{~mL} / \mathrm{kg}$ ) was used as a reference drug. Data represent the relative ear thickness, which was standardized against the previous values before the injection. $N=5$. ${ }^{\#} p<0.01$ $v s$. saline + DW group (Student's $t$-test), $* * p<0.01$ significant $v s$. $P$. acnes + DW group (Dunnett's test).

\subsection{Measurement of Constituents in JHT}

In our previous studies, $Q$. cortex, $S$. spica, and G. radix exhibited strong antioxidant activities in two assays focusing on hydrogen peroxide-dependent oxidation and superoxide anion generation by xanthine oxidase [11]. The results allowed us examine these crude drugs in our dermatitis model. Only Q. cortex and G. radix were evaluated under the protocol previously reported [11], revealing that both crude drugs significantly reduced ear dermatitis by $38 \%$ and $31 \%$ inhibition, respectively, whereas JHT inhibited it by $47 \%$ inhibition. Q. cortex and G. radix appeared to be involved in the antidermatitis efficacies of JHT. We attempted to detect 23 polyphenols originating from the three crude drugs and actually identified 21 polyphenols (Table 1). The quantification analysis showed that liquiritin was the most abundant flavonoid in JHT, and the constituents such as glucoside (liquiritin apioside), aglycon (liquiritigenin), and C-ring-opened analogue (isoliquiritin) showed higher contents compared with the others. Among hydrolysable tannins, 1,2,3,4,6-penta- $O$-galloyl glucose and eugeniin were abundant. The total concentration of the 21 polyphenols was $5.1 \%(w / w)$ in JHT. 
Table 1. Contents of polyphenols in JHT extract.

\begin{tabular}{|c|c|c|c|c|}
\hline Compound Category & Subcategory & Name & Molecular Weight & Amount (mg/g JHT) \\
\hline \multirow{14}{*}{ Flavonoid } & \multirow{3}{*}{ Flavane } & $(+)$-Catechin ${ }^{a}$ & 290.08 & 0.077 \\
\hline & & $(-)$-Epicatechin gallate ${ }^{a}$ & 442.09 & 0.003 \\
\hline & & $( \pm)$-Gallocatechin ${ }^{\text {a }}$ & 306.07 & 0.011 \\
\hline & Flavone & Luteolin $^{a, b}$ & 286.05 & 0.011 \\
\hline & Flavonol & Quercetin ${ }^{a, b}$ & 302.04 & 0.004 \\
\hline & \multirow{5}{*}{ Flavanone } & Hesperidin $^{b}$ & 610.19 & 0.231 \\
\hline & & Hesperetin $^{\mathrm{b}}$ & 302.08 & 0.003 \\
\hline & & Liquiritin $^{\mathrm{c}}$ & 418.13 & 1.872 \\
\hline & & Liquiritin apioside ${ }^{\mathrm{c}}$ & 550.17 & 1.392 \\
\hline & & Liquiritigenin $^{\mathrm{c}}$ & 256.07 & 0.298 \\
\hline & \multirow{3}{*}{ Chalcone } & Isoliquiritin $^{\mathrm{c}}$ & 418.13 & 0.130 \\
\hline & & Isoliquiritin apioside $^{c}$ & 550.17 & 0.167 \\
\hline & & Isoliquiriitgenin ${ }^{c}$ & 256.07 & 0.030 \\
\hline & Isoflavone & Genistein $^{c}$ & 270.05 & 0.002 \\
\hline \multirow{6}{*}{ Hydrolysable tannin } & \multirow{3}{*}{ Gallotannin } & Hamamelitannin $^{\text {a }}$ & 484.09 & 0.024 \\
\hline & & $1,2,3,6$-tetra- $O$-galloyl glucose ${ }^{\text {a }}$ & 788.11 & 0.052 \\
\hline & & $1,2,3,4,6$-penta- $O$-galloyl glucose ${ }^{\text {a }}$ & 940.12 & 0.345 \\
\hline & \multirow{3}{*}{ Ellagitannin } & Eugeniin $^{a}$ & 938.10 & 0.213 \\
\hline & & 1-Desgalloyleugeniin ${ }^{\text {a }}$ & 786.09 & 0.030 \\
\hline & & Castalagin $^{\text {a }}$ & 934.07 & 0.078 \\
\hline Other & Unit of tannin & Gallic acid $^{\text {a }}$ & 170.02 & 0.151 \\
\hline
\end{tabular}

Methanol/purified water extracts of dried JHT (Lot No. 332031900) were analyzed by LC-MS/MS. Compounds marked by ${ }^{\mathrm{a}-\mathrm{c}}$ originated from $Q$. cortex, S. spica, and G. radix, respectively.

This is the first report of a study to measure contents of individual constituents in JHT, showing that JHT has numerous flavonoids and is abundant in catechins with a galloyl moiety and hydrolysable tannins such as gallotannin and ellagitannin. According to the present and previous studies [1,12-14], the tested polyphenols are mainly from the following three crude drugs: Q. cortex, S. spica, and G. radix.

\subsection{Metabolism and Pharmacokinetics of Polyphenols in JHT}

The first step in determining the plasma pharmacokinetics of the polyphenols in JHT was to quantify their intact forms in plasma from JHT-treated rats. Figure 2 shows the plasma concentrations at 1,6 , and $24 \mathrm{~h}$ before and after $\beta$-glucuronidase treatment. Flavonoid concentrations were very low at all time points in non-treated plasma. However, the enzymatic treatment increased concentrations of almost all flavonoids. Liquiritin, liquiritigenin, and genistein appeared as conjugated forms in the plasma and significantly increased from 2.40 to $39.1(1 \mathrm{~h})$, from 0.873 to $200(6 \mathrm{~h})$, and from 0.975 to 78.3 (24 h) ng/mL, respectively. The concentrations of hydrolysable tannins described in Table 1 were below the quantification limits. Gallic acid, a minimal unit of hydrolysable tannins, was not detected in the plasma $(<10 \mathrm{ng} / \mathrm{mL})$. However, 4-O-methylgallic acid, a methylated metabolite of Gallic acid, was quantified in the plasma at $5.57 \mathrm{ng} / \mathrm{mL}(1 \mathrm{~h})$, whereas the concentration was unchanged by $\beta$-glucuronidase treatment. 


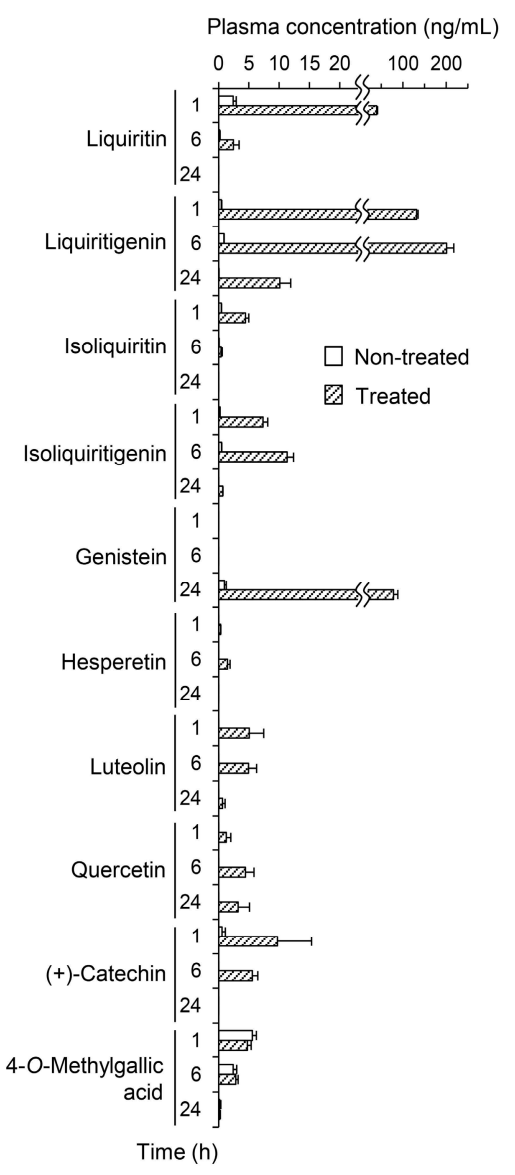

Figure 2. Plasma concentrations of jumihaidokuto (JHT) polyphenols in plasma before and after enzymatic hydrolysis with $\beta$-glucuronidase. Plasma samples were obtained at 1,6 , and $24 \mathrm{~h}$ after JHT administration to rats $(2 \mathrm{~g} / 10 \mathrm{~mL} / \mathrm{kg})$. The polyphenol content in the plasma were measured by LC-MS/MS before and after $\beta$-glucuronidase treatment. Each data point represents the mean \pm S.E. of triplicates.

Focusing on flavonoid glucuronides, liquiritigenin 4'-O-glucuronide (LQG-4'G), liquiritigenin 7-Oglucuronide (LQG-7G), isoliquiritigenin 2'-O-glucuronide (ILQG-2'G), isoliquiritigenin 4'-O-glucuronide (ILQG-4'G), isoliquiritigenin 4-O-glucuronide (ILQG-4G), hesperetin 7- $O$-glucuronide (HPT-7G), luteolin 7-O-glucuronide (LTN-7G), genistein 4'-O-glucuronide (GEN-4'G), genistein 7-O-glucuronide (GEN-7G), and quercetin 3-O-glucuronide (QCN-3G) were examined in another set of animal studies (Figure 3). To the best of our knowledge, our study is the first to determine the overall pharmacokinetics of the glucuronides of liquititigenin and isoliquiritigenin in plasma using standard glucuronides. Various structural changes, such as opening of the C-ring during metabolism, occur in these flavonoids [15]; therefore, it was important to perform pharmacokinetic studies of these compounds. The $C_{\max }$ of LQG-4'G was $84.1 \mathrm{ng} / \mathrm{mL}$, which was more than that of LQG-7G. In addition, the profiles of liquiritigenin glucuronides showed bimodality, and the first peaks appeared very rapidly $(0.25 \mathrm{~h})$ after JHT administration. The profiles of isoliquiritigenin and hesperetin glucuronides showed a time course similar to that of liquitritigenin glucuronides. The profiles may have resulted from the coexistence of aglycones and their glycosides in JHT; the first and second peaks may have been derived from an aglycone and glycoside, respectively. 

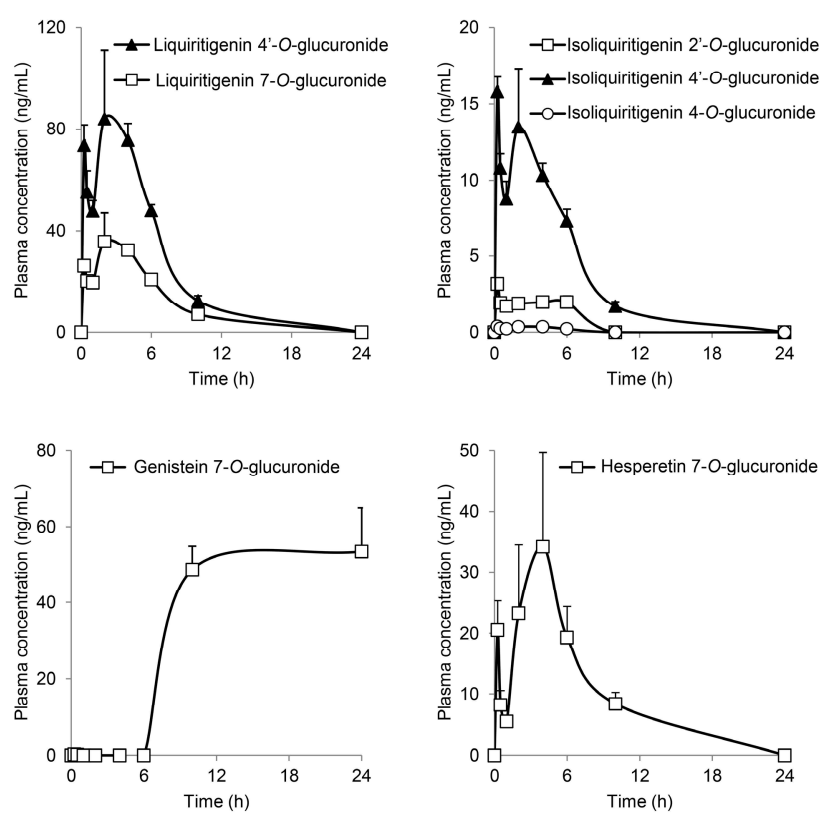

Figure 3. Time course of flavonoid glucuronides in the plasma of JHT-treated rats. Glucuronides of liquiritigenin, isoliquiritigenin, genistein, and hesperetin were measured in the plasma after JHT administration by LC-MS/MS. Each data point represents the mean \pm S.E. of triplicates.

Plasma GEN-7G significantly increased to approximately $50 \mathrm{ng} / \mathrm{mL}$ at 10 and $24 \mathrm{~h}$, but did not start to increase until $6 \mathrm{~h}$ after JHT administration, although genistein is reported to be promptly absorbed into the bloodstream [4]. The phenotype was different from the other flavonoid glucuronides. GEN-4'G, LTN-7G, and QCN-3G did not appear in the plasma during the experiment. Our quantification study of JHT extract showed that JHT contained very little genistein and its glucoside (genistin and sophoricoside) as possible sources of GEN-7G. Thus, we speculated that JHT contains genistein derivatives modified by malonylation, acetylation, and/or another type of glycosylation [16-18]; intestinal bacteria needs time to generate genistein. We will perform further studies to identify the source of GEN-7G. Genistein is subjected to the enterohepatic circulation, resulting in a delayed clearance from the bloodstream [3]. Several investigators have demonstrated that the pharmacokinetics of genistein glucuronides, including GEN-7G, reveals no accumulative properties [4,19].

Table 2 shows the pharmacokinetic parameters of the detected flavonoid glucuronides and 4-O-methylgallic acid.

Table 2. Pharmacokinetic parameters of flavonoid glucuronides and 4-O-methylgallic acid.

\begin{tabular}{ccccc}
\hline Compound & $\boldsymbol{t}_{\mathbf{1} / \mathbf{2}}(\mathbf{h})$ & $\boldsymbol{t}_{\mathbf{m a x}}(\mathbf{H})$ & $\boldsymbol{C}_{\mathbf{m a x}}(\mathbf{n g} / \mathbf{m L})$ & $\mathbf{A U C}_{\mathbf{0}-\text { last }}(\mathbf{n g} \cdot \mathbf{h} / \mathbf{m L})$ \\
\hline Genistein 7- $O$-glucuronide & - & 24.0 & 53.4 & 812 \\
Liquiritigenin 4'-O-glucuronide & 2.27 & 2.00 & 84.1 & 522 \\
Liquiritigenin 7-O-glucuronide & 2.72 & 2.00 & 35.6 & 223 \\
Isoliquiritigenin 2'-O-glucuronide & - & 0.250 & 3.19 & 11.6 \\
Isoliquiritigenin 4'- $O$-glucuronide & 2.25 & 0.250 & 15.8 & 80.8 \\
Isoliquiritigenin 4- $O$-glucuronide & 5.68 & 0.250 & 0.388 & 1.84 \\
Hesperetin 7- $O$-glucuronide & 2.99 & 4.00 & 34.2 & 190 \\
4- $O$-Methylgallic acid & 1.62 & 2.00 & 6.65 & 32.7 \\
\hline
\end{tabular}

-: Not calculated. 


\subsection{Antioxidant Activities of Metabolites of Polyphenols in JHT}

We examined antioxidant activities of the polyphenols and their metabolites appearing in the plasma (Table 3). The IC50 values of GEN-7G, ILQG-4'G, ILQG-2'G, LQG-7G, and HPT-7G were 0.638, $0.691,0.704,0.765$, and $4.27 \mu \mathrm{g} / \mathrm{mL}$, respectively, whereas LQG-4'G and 4-O-methylgallic acid were not detectable even at the highest concentration. Only 4-O-methylgallic acid, but not all flavonoid glucuronides, weakened in the nitric oxide reaction, with an $\mathrm{IC}_{50}$ of $3.59 \mu \mathrm{g} / \mathrm{mL}$. In addition, glycyrrhetic acid, a metabolite of glycyrrhizic acid originating from G. radix, and cimifugin from Saposhnikoviae radix ( $S$. radix), are easily absorbed into the bloodstream $[1,20]$. In the rat plasma used in the present study, we actually confirmed that glycyrrhetic acid and cimifugin were identified by major peaks (Supplementary Figure S1). The two compounds were also evaluated as reference compounds of non-polyphenols. Consequently, both showed no activity.

Table 3. Antioxidant activity of metabolites absorbed in plasma.

\begin{tabular}{ccc}
\hline & \multicolumn{2}{c}{ Antioxidant activity, $\mathbf{I C}_{\mathbf{5 0}}(\boldsymbol{\mu g} / \mathbf{m L})$} \\
\cline { 2 - 3 } & Hydrogen Peroxide & Nitric Oxide \\
\hline Genistein 7- $O$-glucuronide & 0.638 & n.d. \\
Liquiritigenin 4'- $O$-glucuronide & n.d. & n.d. \\
Liquiritigenin 7-O-glucuronide & 0.765 & n.d. \\
Isoliquiritigenin 2'-O-glucuronide & 0.704 & n.d. \\
Isoliquiritigenin 4'-O-glucuronide & 0.691 & n.d. \\
Hesperetin 7- $O$-glucuronide & 4.27 & n.d. \\
4- $O$-Methylgallic acid & n.d. & 3.59 \\
\hline Glycyrrhetic acid & n.d. & n.d. \\
Cimifugin & n.d. & n.d. \\
\hline
\end{tabular}

All samples were evaluated at $0.1,0.3,1,3,10$, or $30 \mu \mathrm{mol} / \mathrm{L} . N=3$. The $\mathrm{IC}_{50}$ values were calculated from curves constructed by plotting antioxidant activity (\%) vs. test sample concentration. Data are shown as $\mathrm{IC}_{50}(\mu \mathrm{g} / \mathrm{mL})$. n.d.: Not detectable at the maximal concentration $(30 \mu \mathrm{mol} / \mathrm{L})$. Namely, $4-O$-methylgallic acid, $5.52 \mu \mathrm{g} / \mathrm{mL}$; Hesperetin 7-O-glucuronide, $14.4 \mu \mathrm{g} / \mathrm{mL}$; Genistein 7-O-glucuronide, $13.4 \mu \mathrm{g} / \mathrm{mL}$; Liquiritigenin 7-O-glucuronide; Liquiritigenin 4'-O-glucuronide; Isoliquiritigenin 2'-O-glucuronide; Isoliquiritigenin 4'-O-glucuronide, $13.0 \mu \mathrm{g} / \mathrm{mL}$; Glycyrrhetic acid, $14.1 \mu \mathrm{g} / \mathrm{mL}$; and Cimifugin, $9.19 \mu \mathrm{g} / \mathrm{mL}$.

We finally tested whether there was a difference between conjugates and aglycone to understand how much the glucuronidation process affects antioxidant activities. The results showed that anti-ROS activities of LQG-7G, ILQG-4'G, and GEN-7G were completely identical to that of their aglycones (Figure 4). However, HPT-7G concentration was partially decreased and LQG-4'G showed no activity.

The chemical structures of the metabolites in Table 2 are shown in Figure 5. According to the studies on structure-activity correlations in flavonoids [2,21,22], the hydroxyl group of C4'-position of the B-ring is critical for expression of antioxidant and antiradical activities, and glucuronidation of the C4'-position remarkably diminishes those activities. In contrast, glucuronidation of the $\mathrm{C} 7$ position of the A-ring does not affect it. In our study, liquiritigenin, which showed strong activity, was deactivated by glucuronidation of the $\mathrm{C}^{\prime}$ position (LQG-4'G), but not the $\mathrm{C} 7$ position (LQG-7G). Isoliquiritigenin and genistein were also unchanged by glucuronidation to the A-ring. 

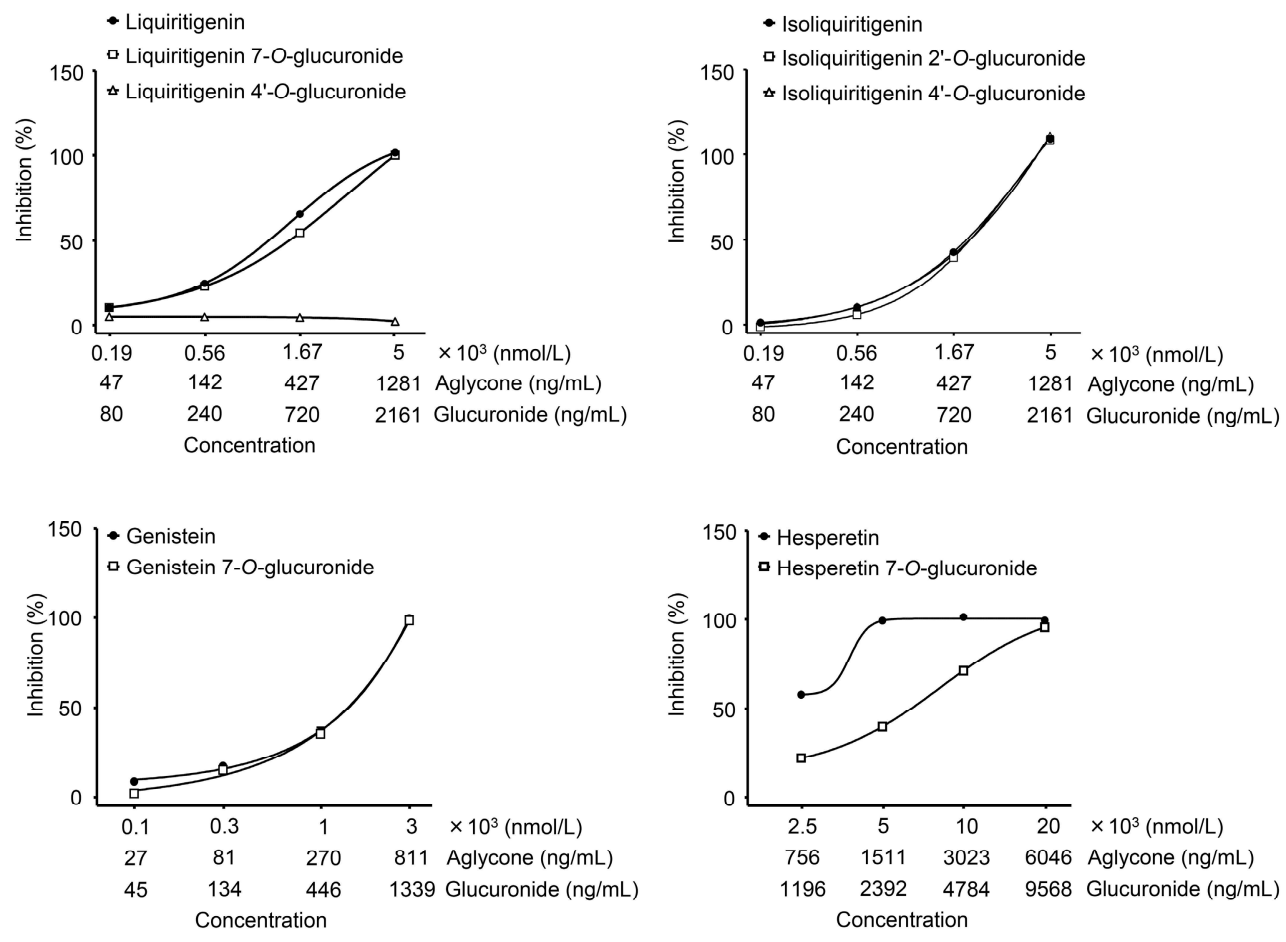

Figure 4. Concentration dependency of flavonoid glucuronides and comparison with aglycones. Glucuronides and aglycones of flavonoid were assayed at serially-diluted concentrations to assess on hydrogen peroxide-dependent oxidation. Test samples were incubated with hydrogen peroxide for 5 min, followed by mixing with a probe of oxidant activity. After $15 \mathrm{~min}$ incubation, the hydrogen peroxide-dependent fluorescent intensity was measured. Data are shown as mean \pm S.E. of triplicates.
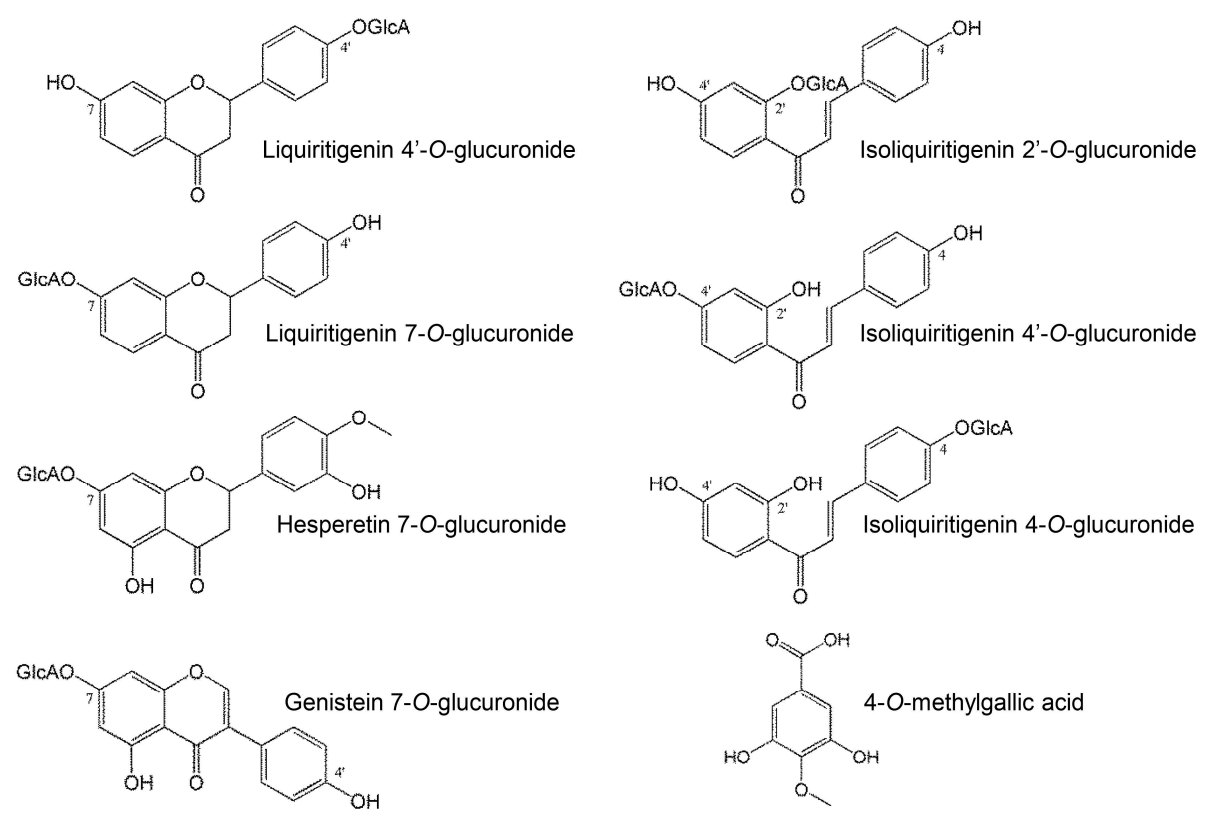

Figure 5. Chemical structures of metabolites appearing in plasma.

Although LQG-4'G showed the highest concentration of all of the glucuronides in the plasma, it exerted no activity. Oral administration of liquiritigenin was reported to inhibit carrageenan-induced 
edema in an animal model of acute inflammation [23]. Therefore, a metabolite from liquiritigenin (LQG-7G) may contribute to the antidermatitis effect of JHT. GEN-7G was thought to be the most potent on effects of JHT because it showed a high concentration in the plasma and strong anti-ROS activity. GEN-7G was absorbed at $C_{\max }: 53.4 \mathrm{ng} / \mathrm{mL}, t_{\max }: 24 \mathrm{~h}$. The IC50 in anti-ROS activity was $638 \mathrm{ng} / \mathrm{mL}$. A statistically significant activity (25\% inhibition) was observed at $134 \mathrm{ng} / \mathrm{mL}$, as shown in Figure 4. There were no significant differences between the $C_{\max }$ and bioactive concentrations in vitro. It is easy to imagine that many other metabolites and intact constituents including unknowns may enter the bloodstream. It is important to consider efficacies of JHT as a whole in combination with individual effects of JHT metabolites.

As reported previously, tannins were minimally absorbed into the bloodstream [2,24]. Tannins in JHT were not detected in the plasma, although there was a problem with their detection sensitivity. Orally administered gallotannin and ellagitannin are hydrolyzed by tannases in intestinal bacteria, resulting in significant generation of Gallic acid and/or ellagic acid [7,24]. Gallic acid is metabolized mainly to 4-O-methylgallic acid in rats [25]. Therefore, our study used 4-O-methylgallic acid and demonstrated its antioxidant activity. Several studies have reported that oral administration of Gallic acid evoked various benefits including anti-inflammatory, antinociceptive, and antiedematogenic effects and that 4-O-methylgallic acid has various biological activities [8,26-28]. Gallic acid metabolites 4-O-methylgallic acid, 3-O-methylgallic acid, and 3,4-O-dimethylgallic acid were reported to be identified in the urine of volunteers who ingested black tea [29]. We examined the potential of 3-O-methylgallic acid in antioxidant assays, and it showed a strong activity against ROS but not nitric oxide (data not shown). More emphasis is needed on the pharmacological aspect of Gallic acid and its derivatives.

\subsection{Pharmacological Characteristics of JHT Showing Antioxidant Effects}

ROS are formed constitutively by mitochondria and NADPH oxidase, and their production is augmented by inflammation. It has been recently reported that ROS upregulates caspase expression and activation of the Nod-like receptor protein 3 inflammasome during inflammation [30]. Nitric oxide is produced by nitric oxide synthase, and it plays various biological roles such as neurotransmitter and vascular tone controller through activation of guanylate cyclase. In the case of an infectious response, phagocytic cells such as neutrophils produce large quantities of ROS and reactive nitrogen species (RNS) to kill foreign microbes. Excessive release of extracellular ROS/RNS damages surrounding healthy cells, and large numbers of dead neutrophils and cellular debris can cause secondary inflammation [31,32]. The pathophysiological roles of radical molecules have been well studied in models of ischemiareperfusion injury [33,34]. This implies that ROS/RNS are critical to upregulate microvascular circulation in order to increase blood flow during an emergency following a decrease in oxygen concentration. As reported previously [35], a histological analysis of inflamed ears injected with $P$. acnes showed evidence of abscesses in which numerous neutrophils were accumulated (data not shown) $24 \mathrm{~h}$ after the bacterial injection. It is plausible that excessive release of ROS/RNS occurred at the inflamed ears due to P. acnes injection, followed by damage of vessel endothelial cells and vasodilation. Local ROS/RNS may have been significantly involved in $P$. acnes-induced ear thickness and rubefaction, although other inflammatory factors such as histamines and bioactive lipids were also involved. In the models of dermatitis caused by UV-irradiation, increased radical molecules directly trigger signs of acute inflammation such as skin 
edema and rubefaction [36]. Antioxidant agents, including ascorbic acid and botanical flavonoids, have been reported to prevent skin inflammation induced by UV-irradiation [37-40].

JHT contains flavonoid-rich crude drugs and tannin-rich one. Flavonoids inhibited ROS preferentially, and only 4-O-methylgallic acid inhibited the nitric oxide reaction. These findings are of interest when discussing the multipotency of the antioxidant effects of JHT. In addition, flavonoid glucuronides rarely function via direct modifications of intracellular events (such as kinase cascades and estrogen receptor activation) because glucuronides do not generally pass through cell membranes consisting of a lipid bilayer; they function mainly at extracellular sites. It is reasonable to assume that JHT suppresses only extracellular and excessive toxic radicals.

\section{Experimental Section}

\subsection{JHT and Test Compounds}

JHT powder (Lot No. 332031900), the base powder of JHT without excipients, was obtained from Tsumura \& Co. (Tokyo, Japan), which manufactures it as an aqueous extract mixture of 10 crude components (percent composition shown in parentheses): Platycodi radix (14.3\%), Bupleuri radix (14.3\%), Cnidii rhizoma (14.3\%), Poria sclerotium (14.3\%), Q. cortex (14.3\%), Araliae cardatae rhizoma (7.1\%), S. radix (7.1\%), G. radix (4.8\%), S. spica (4.8\%), and Zingiberis rhizoma (4.8\%).

As candidate compounds of JHT, liquiritin apioside, isoliquiritin, isoliquiritin apioside, isoliquiritigenin, luteolin, genistin, (+)-catechin, (-)-epicatechin gallate, 1,2,3,6-tetra- $O$-galloyl glucose, 1,2,3,4,6-penta$O$-galloyl glucose, eugeniin, 1-desgalloyl eugeniin, castalagin, LQG-4'G, LQG-7G, ILQG-2'G, ILQG-4'G, ILQG-4G, glycyrrhetic acid, and cimifugin were supplied by Tsumura \& Co. with purities high enough to be evaluated in biological tests. The following compounds were purchased from chemical manufacturers: liquiritin, liquiritigenin, genistein, hesperidin, hesperetin, quercetin, (-)-gallocatechin, and Gallic acid from Wako Pure Chemical Industries (Osaka, Japan), GEN-4'G, GEN-7G, and HPT-7G from Toronto Research Chemical Industries (Toronto, ON, Canada), 4-O-methylgallic acid from ChromaDex (Irvine, CA, USA), sophoricoside from AK Scientific (Union City, CA, USA), LTN-7G from Chengdu Biopurify Phytochemicals Co. (Sichuan, China), QCN-3G from WuXi AppTec Co. (Shanghai, China), and hamamelitannin from Sigma-Aldrich (St. Louis, MO, USA).

\subsection{Animals}

Male Sprague Dawley rats were purchased from Japan SLC (Shizuoka, Japan) and used at the age of 7-8 weeks. The rats were allowed free access to water and standard laboratory food and housed at a temperature of $23 \pm 3{ }^{\circ} \mathrm{C}$, relative humidity of $50 \% \pm 20 \%$, and a 12-h light:12-h dark cycle, with lights on from 7:00 a.m. to 7:00 p.m. daily. All experimental procedures were performed according to the "Guidelines for the Care and Use of Laboratory Animals" of Tsumura \& Co. Ethical approval of the experimental procedures was obtained from the Laboratory Animal Committee of Tsumura \& Co.

\subsection{P. acnes-Induced Acute Dermatitis and Measurement of Ear Thickness}

The $P$. acnes strain ATCC6919 was purchased from Microbiologics Inc. (St. Cloud, MN, USA) and cultured in GAM broth (Nissui Pharmaceutical Co., Tokyo, Japan) in an anaerobic atmosphere using 
a GasPak system (Mitsubishi Gas Chemical Co., Tokyo, Japan). To prepare the P. acnes suspension for dermatitis experiments in rat ears, $P$. acnes was harvested, washed with phosphate-buffered saline (PBS), and centrifuged at 10,000 $\mathrm{g}$ for $10 \mathrm{~min}$. After washing twice with PBS, P. acnes was heat-killed at $95{ }^{\circ} \mathrm{C}$ for $5 \mathrm{~min}$, freeze-dried, and stored at $-80{ }^{\circ} \mathrm{C}$ until use.

$P$. acnes-induced dermatitis was induced using the procedure described previously with a minor modification [35]. Heat-killed $P$. acnes $(0.14 \mathrm{mg}$ in $50 \mu \mathrm{L}$ saline) or saline (control) was intradermally injected into the ventral side of both ears using a microsyringe (Hamilton Co., Reno, NV, USA) under isoflurane anesthesia. JHT was orally administered to rats at $0.5 \mathrm{~g} / \mathrm{kg}$ in distilled water $1 \mathrm{~h}$ before and $6 \mathrm{~h}$ after the bacterial injection. PDN (Shionogi Pharmaceutical Co., Osaka, Japan) was orally administered to rats at a dose of $10 \mathrm{mg} / \mathrm{kg}$ in distilled water. The ear thickness was measured using a dial thickness gauge micrometer (Ozaki MFG Co., Tokyo, Japan) at 0 and $24 \mathrm{~h}$ after the injection. All data on the increase in ear thickness were expressed as a percentage of the previous value in each individual rat, and as the mean \pm S.E. Statistical significance was evaluated by one-way analysis of variance, followed by the Dunnett's multiple comparisons or unpaired Student's $t$-test. A $p$-value of $<0.05$ was considered significant.

\subsection{Measurement of Constituents in JHT by LC-MS/MS}

Preparation of analytical samples and composition of LC-MS/MS systems were described in the previous report [12], i.e., the constituents were extracted twice from $100 \mathrm{mg}$ of JHT powder by $4 \mathrm{~mL}$ of methanol/purified water $(75: 25, v / v)$ and $4 \mathrm{~mL}$ of methanol/purified water $(50: 50, v / v)$ at sequential two-step. The extract solution, undiluted or diluted 10- or 100-fold with methanol, was injected into the two LC-MS/MS systems after pooling with a respective internal standard solution, niflumic acid (Sigma-Aldrich) or vincamine (Tokyo Chemical Industry Co., Tokyo, Japan). Analytical conditions are summarized in Supplementary Tables S1 and S2.

\subsection{Pharmacokinetic Analysis of Constituents in JHT and Metabolites by LC-MS/MS}

JHT prepared in water was orally administered to 16-h fasted rats weighing 230-285 $\mathrm{g}$ at a dose of $2 \mathrm{~g} / 10 \mathrm{~mL} / \mathrm{kg}$ ( $n=3 \mathrm{each})$. The higher dose than that in the pharmacological test was used to detect more constituents. Blood was withdrawn from the abdominal inferior vena cava with a heparinized syringe at $0.25,0.5,1,2,4,6,10$, or $24 \mathrm{~h}$ after the administration. Plasma was obtained by centrifugation at $1700 \mathrm{~g}$ for $15 \mathrm{~min}$ at $4{ }^{\circ} \mathrm{C}$ and stored at $-80{ }^{\circ} \mathrm{C}$ until use. In some experiments to deconjugate glucuronidated polyphenols, the samples were incubated with $75 \mathrm{mmol} / \mathrm{L}$ phosphate buffer containing 200 units/mL $\beta$-glucuronidase from Escherichia coli (Sigma-Aldrich) for $2 \mathrm{~h}$ at $37^{\circ} \mathrm{C}$.

For quantification of liquiritin, liquiritigenin, isoliquiritin, isoliquiritigeniin, hesperidin, hesperetin, castalagin, and all flavonoid glucuronides, $25-60 \mu \mathrm{L}$ of standard solution and $25 \mu \mathrm{L}$ of internal standard solution were added to $200-300 \mu \mathrm{L}$ of plasma samples, followed by mixing well. Methanol $(750-800 \mu \mathrm{L})$ was added to the solutions, followed by mixing and centrifugation ( $7000 \mathrm{~g}, 5 \mathrm{~min})$. The supernatants were collected and dried at $40^{\circ} \mathrm{C}$ under a stream of nitrogen gas. Then, the dried residue was dissolved in $50-100 \mu \mathrm{L}$ of the HPLC mobile phase of each analytical method, and a 1-20 $\mu \mathrm{L}$ portion was injected into the LC-MS/MS systems. For quantification of genistein, luteolin, quercetin, $(+)$-catechin, $(-)$-epicatechin gallate, $( \pm$ )-gallocatechin, Gallic acid, 4- $O$-methylgallic acid, hamamelitannin, 
1,2,3,6-tetra- $O$-galloyl glucose, 1,2,3,4,6-penta- $O$-galloyl glucose, eugeniin, and 1-desgalloyl eugeniin, $200 \mu \mathrm{L}$ of plasma samples were used. The amounts were measured using the same procedure, as described previously, except ethyl acetate $(500 \mu \mathrm{L})$ and $2 \%$ acetic acid $(100 \mu \mathrm{L})$, instead of methanol, were used concurrently as an extraction solution.

Plasma pharmacokinetic data were analyzed by non-compartmental modeling using Phoenix WinNonlin (version 6.3, Certara L.P., St. Louis, MO, USA) to determine various pharmacokinetic constants including the maximum concentration $\left(C_{\max }\right)$, time to maximum concentration $\left(t_{\max }\right)$, apparent elimination half-life $\left(t_{1 / 2}\right)$, and area under the plasma concentration-time curve from time zero to the last observation ( $\mathrm{AUC}_{0-\text { last }}$ ). The $t_{1 / 2}$ was divided by $\log _{\mathrm{e}} 2 / k \mathrm{e}$, where $k \mathrm{e}$ is the terminal elimination (at least three data points on the descending linear limb) rate constant.

\subsection{Antioxidant Assays}

To assess ROS, OxiSelect In vitro ROS/RNS Assay kits (R \& D Biosystems, Minneapolis, MN, USA) were used according to manufacturer's instructions. Briefly, a test sample was incubated with $2.5 \mu \mathrm{mol} / \mathrm{L}$ of hydrogen peroxide for $5 \mathrm{~min}$, and dichlorodihydrofluorescin DiOxyQ (DCFH-DiOxyQ) was added as a probe of oxidant activity. After $15 \mathrm{~min}$ incubation, the hydrogen peroxide-dependent fluorescent intensity was measured using a fluorescent plate reader (Infinite M200; Tecan, Wako Pure Chemical Industries). Percentage of inhibition of ROS was calculated using the following equation:

$$
\left[1-\left(\text { sample } \mathrm{RFU}^{\mathrm{a}}-\text { base } \mathrm{RFU}^{\mathrm{b}}\right) /\left(\text { control } \mathrm{RFU}^{\mathrm{c}}-\text { base } \mathrm{RFU}^{\mathrm{b}}\right)\right] \times 100
$$

where RFU is a relative fluorescent unit of reaction solution, a is RFU of DCFH-DiOxyQ with hydrogen peroxide and sample, $b$ is RFU of DCFH-DiOxyQ alone, and c is RFU of DCFH-DiOxyQ with hydrogen peroxide.

To quantify nitric oxide, a test sample was incubated at $37{ }^{\circ} \mathrm{C}$ for $150 \mathrm{~min}$ in PBS supplemented with $30 \mu \mathrm{mol} / \mathrm{L}$ of $( \pm)-(E)$-4-ethyl-2-[(E)-hydroxyimino]-5-nitro-3-hexenamide (NOR3). Nitric oxide generated naturally from NOR3, was measured using the Griess reagent ( $1 \%$ sulfanilamide, $0.1 \%$ $N$-(1-naphthyl) ethylenediamine dihydrochlride in 5\% $\left.\mathrm{H}_{3} \mathrm{PO}_{4}\right)$. Briefly, $100 \mu \mathrm{L}$ of the reacted solution was incubated for $5 \mathrm{~min}$ with $100 \mu \mathrm{L}$ Griess reagent. Optical densities at $540 \mathrm{~nm}$ were then measured.

\section{Conclusions}

In this study, we conducted the following: (1) identification of polyphenols in JHT; (2) pharmacokinetic analysis of the polyphenols and metabolites; and (3) biological evaluation of the absorbed metabolites in assays associated with the development of dermatitis to identify active metabolites of JHT. JHT was found to contain various polyphenols that appeared to inhibit dermal inflammation via antioxidant effects of their metabolites, including GEN-7G and LQG-7G. GEN-7G is an active metabolite of JHT, based on a comparison of the absorbed concentrations and range of bioactive concentrations. Our results contribute to an understanding of the mechanisms of oral polyphenols, crude drugs, and diets containing beneficial polyphenols. 


\section{Supplementary Materials}

Supplementary materials can be accessed at: http:/www.mdpi.com/1420-3049/20/10/18031/s1.

\section{Acknowledgments}

We are deeply grateful to our colleague Chiharu Sadakane for helpful discussion. This study was funded by Tsumura \& Co.

\section{Author Contributions}

T. Matsumoto performed experiments, analyzed data, prepared figures and tables, interpreted results of experiments, and drafted the manuscript; Y. Matsubara, Y. Mizuhara, K. Sekiguchi, J. Koseki, and K. Tsuchiya performed experiments and analyzed data; H. Nishimura and J. Watanabe provided constitutive and pharmacokinetic information and interpreted results of experiments; K. Maemura, T. Hattori, and Y. Kase interpreted results of experiments; and A. Kaneko interpreted results of experiments and revised the manuscript.

\section{Conflicts of Interest}

The authors declare no conflict of interest.

\section{References}

1. Asl, M.N.; Hosseinzadeh, H. Review of pharmacological effects of glycyrrhiza sp. And its bioactive compounds. Phytother. Res. 2008, 22, 709-724.

2. Harada, M.; Kan, Y.; Naoki, H.; Fukui, Y.; Kageyama, N.; Nakai, M.; Miki, W.; Kiso, Y. Identification of the major antioxidative metabolites in biological fluids of the rat with ingested (+)-catechin and (-)-epicatechin. Biosci. Biotechnol. Biochem. 1999, 63, 973-977.

3. Sfakianos, J.; Coward, L.; Kirk, M.; Barnes, S. Intestinal uptake and biliary excretion of the isoflavone genistein in rats. J. Nutr. 1997, 127, 1260-1268.

4. Soucy, N.V.; Parkinson, H.D.; Sochaski, M.A.; Borghoff, S.J. Kinetics of genistein and its conjugated metabolites in pregnant sprague-dawley rats following single and repeated genistein administration. Toxicol. Sci. 2006, 90, 230-240.

5. Franke, A.A.; Lai, J.F.; Halm, B.M. Absorption, distribution, metabolism, and excretion of isoflavonoids after soy intake. Arch. Biochem. Biophys. 2014, 559, 24-28.

6. Hayashi, T.; Ueda, S.; Tsuruta, H.; Kuwahara, H.; Osawa, R. Complexing of green tea catechins with food constituents and degradation of the complexes by lactobacillus plantarum. Biosci. Microbiota Food Health 2012, 31, 27-36.

7. Lopez de Felipe, F.; de Las Rivas, B.; Munoz, R. Bioactive compounds produced by gut microbial tannase: Implications for colorectal cancer development. Front. Microbiol. 2014, 5, doi:10.3389/fmicb.2014.00684. 
8. Na, H.J.; Lee, G.; Oh, H.Y.; Jeon, K.S.; Kwon, H.J.; Ha, K.S.; Lee, H.; Kwon, Y.G.; Kim, Y.M. 4-O-methylgallic acid suppresses inflammation-associated gene expression by inhibition of redox-based nf-kappab activation. Int. Immunopharmacol. 2006, 6, 1597-1608.

9. Higaki, S.; Toyomoto, T.; Morohashi, M. Seijo-bofu-to, jumi-haidoku-to and toki-shakuyaku-san suppress rashes and incidental symptoms in acne patients. Drugs Exp. Clin. Res. 2002, 28, 193-196.

10. Nose, M.; Sakushima, J.; Harada, D.; Ogihara, Y. Comparison of immunopharmacological actions of 8 kinds of kampo-hozais clinically used in atopic dermatitis on delayed-type hypersensitivity in mice. Biol. Pharm. Bull. 1999, 22, 48-54.

11. Matsubara, Y.; Sekiguchi, K.; Koseki, J.; Tsuchiya, K.; Kaneko, A.; Hattori, T.; Kase, Y. Preventive effect of a traditional japanese medicine, jumihaidokuto, in animal models of acute dermatitis. Jpn. Pharmacol. Ther. 2015, 43, 791-798.

12. Koseki, J.; Matsumoto, T.; Matsubara, Y.; Tsuchiya, K.; Mizuhara, Y.; Sekiguchi, K.; Nishimura, H.; Watanabe, J.; Kaneko, A.; Hattori, T.; et al. Inhibition of rat $5 \alpha$-reductase activity and testosterone-induced sebum synthesis in hamster sebocytes by an extract of quercus acutissima cortex. eCAM 2015, 2015, doi:10.1155/2015/853846.

13. Zeng, X.L.; Fu, G.M.; Tian, K.; Sun, J.X.; Xiong, H.B.; Huang, X.Z.; Jiang, Z.Y. Acutissimanide, a new lignan with antioxidant activity isolated from the bark of Quercus acutissima carruth. Nat. Prod. Res. 2014, 28, 1364-1370.

14. Wang, B.S.; Huang, G.J.; Tai, H.M.; Huang, M.H. Antioxidant and anti-inflammatory activities of aqueous extracts of schizonepeta tenuifolia briq. Food Chem. Toxicol. 2012, 50, 526-531.

15. Cuendet, M.; Guo, J.; Luo, Y.; Chen, S.; Oteham, C.P.; Moon, R.C.; van Breemen, R.B.; Marler, L.E.; Pezzuto, J.M. Cancer chemopreventive activity and metabolism of isoliquiritigenin, a compound found in licorice. Cancer Prev. Res. 2010, 3, 221-232.

16. Caligiani, A.; Palla, G.; Maietti, A.; Cirlini, M.; Brandolini, V. 1H NMR fingerprinting of soybean extracts, with emphasis on identification and quantification of isoflavones. Nutrients 2010, 2 , 280-289.

17. Fang, C.; Wan, X.; Tan, H.; Jiang, C. Identification of isoflavonoids in several kudzu samples by high-performance liquid chromatography coupled with electrospray ionization tandem mass spectrometry. J. Chromatogr. Sci. 2006, 44, 57-63.

18. Song, T.; Barua, K.; Buseman, G.; Murphy, P.A. Soy isoflavone analysis: Quality control and a new internal standard. Am. J. Clin. Nutr. 1998, 68, 1474S-1479S.

19. Shelnutt, S.R.; Cimino, C.O.; Wiggins, P.A.; Ronis, M.J.; Badger, T.M. Pharmacokinetics of the glucuronide and sulfate conjugates of genistein and daidzein in men and women after consumption of a soy beverage. Am. J. Clin. Nutr. 2002, 76, 588-594.

20. Li, Y.; Zhao, L.; Zhang, H.; Jia, J.; Lv, L.; Zhou, G.; Chai, Y.; Zhang, G. Comparative pharmacokinetics of prim- $O$-glucosylcimifugin and cimifugin by liquid chromatography-mass spectrometry after oral administration of radix saposhnikoviae extract, cimifugin monomer solution and prim-O-glucosylcimifugin monomer solution to rats. Biomed. Chromatogr. 2012, 26, 1234-1240.

21. Burda, S.; Oleszek, W. Antioxidant and antiradical activities of flavonoids. J. Agric. Food Chem. 2001, 49, 2774-2779. 
22. Moon, J.H.; Tsushida, T.; Nakahara, K.; Terao, J. Identification of quercetin 3-O- $\beta$-D-glucuronide as an antioxidative metabolite in rat plasma after oral administration of quercetin. Free Radic. Biol. Med. 2001, 30, 1274-1285.

23. Kim, Y.W.; Zhao, R.J.; Park, S.J.; Lee, J.R.; Cho, I.J.; Yang, C.H.; Kim, S.G.; Kim, S.C. Anti-inflammatory effects of liquiritigenin as a consequence of the inhibition of NF- $\kappa \mathrm{B}$-dependent iNOS and proinflammatory cytokines production. Br. J. Pharmacol 2008, 154, 165-173.

24. Zhang, J.; Li, L.; Kim, S.H.; Hagerman, A.E.; Lu, J. Anti-cancer, anti-diabetic and other pharmacologic and biological activities of penta-galloyl-glucose. Pharm. Res. 2009, 26, 2066-2080.

25. Zong, L.; Inoue, M.; Nose, M.; Kojima, K.; Sakaguchi, N.; Isuzugawa, K.; Takeda, T.; Ogihara, Y. Metabolic fate of Gallic acid orally administered to rats. Biol. Pharm. Bull. 1999, 22, 326-329.

26. Jeon, K.S.; Na, H.J.; Kim, Y.M.; Kwon, H.J. Antiangiogenic activity of 4-O-methylgallic acid from Canavalia gladiata, a dietary legume. Biochem. Biophys. Res. Commun. 2005, 330, 1268-1274.

27. Trevisan, G.; Rossato, M.F.; Tonello, R.; Hoffmeister, C.; Klafke, J.Z.; Rosa, F.; Pinheiro, K.V.; Pinheiro, F.V.; Boligon, A.A.; Athayde, M.L.; et al. Gallic acid functions as a trpal antagonist with relevant antinociceptive and antiedematogenic effects in mice. Naunyn. Schmiedeberg's Arch. Pharmacol. 2014, 387, 679-689.

28. Maurya, H.; Mangal, V.; Gandhi, S.; Prabhu, K.; Ponnudurai, K. Prophylactic antioxidant potential of Gallic acid in murine model of sepsis. Int. J. Inflamm. 2014, 2014, doi:10.1155/2014/580320.

29. Hodgson, J.M.; Morton, L.W.; Puddey, I.B.; Beilin, L.J.; Croft, K.D. Gallic acid metabolites are markers of black tea intake in humans. J. Agric. Food Chem. 2000, 48, 2276-2280.

30. Harijith, A.; Ebenezer, D.L.; Natarajan, V. Reactive oxygen species at the crossroads of inflammasome and inflammation. Front. Physiol. 2014, 5, 352.

31. Kono, H.; Onda, A.; Yanagida, T. Molecular determinants of sterile inflammation. Curr. Opin. Immunol. 2014, 26, 147-156.

32. Brune, B.; Dehne, N.; Grossmann, N.; Jung, M.; Namgaladze, D.; Schmid, T.; von Knethen, A.; Weigert, A. Redox control of inflammation in macrophages. Antioxid. Redox Signal. 2013, 19, 595-637.

33. Ferrari, R.S.; Andrade, C.F. Oxidative stress and lung ischemia-reperfusion injury. Oxid. Med. Cell Longev. 2015, 2015, doi:10.1155/2015/590987.

34. Malek, M.; Nematbakhsh, M. Renal ischemia/reperfusion injury; from pathophysiology to treatment. J. Renal Injury Prev. 2015, 4, 20-27.

35. De Young, L.M.; Young, J.M.; Ballaron, S.J.; Spires, D.A.; Puhvel, S.M. Intradermal injection of Propionibacterium acnes: A model of inflammation relevant to acne. J. Investig. Dermatol. 1984, 83, 394-398.

36. Mio, M.; Yabuta, M.; Kamei, C. Ultraviolet B (UVB) light-induced histamine release from rat peritoneal mast cells and its augmentation by certain phenothiazine compounds. Immunopharmacology 1999, 41, 55-63.

37. Goto, Y.; Watanabe, N.; Kogawa, N.; Tsuchiya, M.; Takahashi, O.; Uchi, H.; Furue, M.; Hayashi, H. Cx-659s: A novel diaminouracil derivative that has antioxidative and acute anti-inflammatory activities. Eur. J. Pharmacol. 2002, 438, 189-196. 
38. Iovine, B.; Iannella, M.L.; Gasparri, F.; Giannini, V.; Monfrecola, G.; Bevilacqua, M.A. A comparative analysis of the photo-protective effects of soy isoflavones in their aglycone and glucoside forms. Int. J. Mol. Sci. 2012, 13, 16444-16456.

39. Afaq, F.; Katiyar, S.K. Polyphenols: Skin photoprotection and inhibition of photocarcinogenesis. Mini Rev Med. Chem. 2011, 11, 1200-1215.

40. Masaki, H. Role of antioxidants in the skin: Anti-aging effects. J. Dermatol. Sci. 2010, 58, 85-90.

Sample Availability: Not available.

(C) 2015 by the authors; licensee MDPI, Basel, Switzerland. This article is an open access article distributed under the terms and conditions of the Creative Commons Attribution license (http://creativecommons.org/licenses/by/4.0/). 